
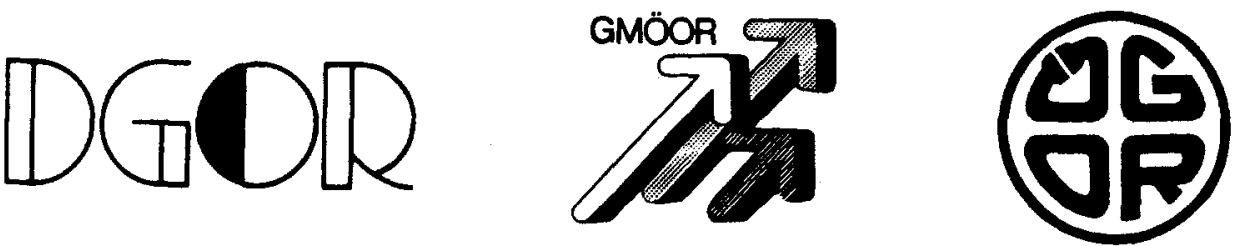

\title{
SYMPOSIUM ON OPERATIONS RESEARCH 1995
}

\author{
Jahrestagung der DGOR, GMÖOR und ÖGOR (SOR '95) \\ Universität Passau, 13. September - 15. September, 1995
}

Soktion
1
2
3
4
5
6
7
8
9
10
11
12
13
14
15
16
17
18
19
20
21

Lineare Optimierung
Nichtlineare Optimlerung
Kombinatorlsche und Diskrete Optimierung
Stochastische Modelle und Optlmierung
Echtzelt-Optimlerung
Schedulling
Kontrolltheorle
Statistik. Okonometrie
Makrookonomie
Mathematische Ökonomle und Spleltheorie
Neuronale Netze und Fuzzy Control
Simulation
Entscheldungsunterstützende Systeme und Informatlonssysteme
Banken, Finanzlerung. Verslcherungen
Fertigungswirtschaft
Logistik
Transport und Verkehr
Lagerhaltung
Anwendungsberichte aus der Praxis
Entscheldungstheorie und experimentelle Wirtschaftsforschung
Aspekte des Umweltschutzes

Sektlonslelter

Borgwardt

Kummer

Reinelt

Rleder

Bock

Kámpke

Tröltzsch

Frledmann

R. Rlchter

Trockel

Weber

Eschenbacher

Solte

Loistl

Ziegler

Vahrenkamp

sahling

Inderfurth

Schuster

Leopold-Wlldburger

Haasis

Tagungssprachen sind Englisch und Deutsch. Das wissenschaftllche Programm enthălt eingeladene Plenar- und Parallelvorträge sowie Sektionsvorträge. Die Zelt für dle Sektionsvorträge einschlleßlich Dlskussion ist auf 30 Minuten beschränkt.

Letzter Anmeldetermin für Abstracts ist der 1. April, 1995.

Präsentation von Software: Es wird begrüBt, wenn die Tellnehmer Softwarelösungen zu ihren Belträgen oder Softwaresysteme vorstellen. Letzter Termin für die vorläuflige Anmeldung zur Tellnahme ist der 1. Februar, 1995.

\section{Organlsationskomiteo:}

Prof. Dr. P. Kleinschmidt Prof. Dr. H. Schmalen Prof. Dr. B. Schmidt Prof. Dr. W. Schweltzer Prof. Dr. J. Wilhelm Prof. Dr. H. Zlegler

\section{Programmkomlteo:}

Prof. Dr. P. Kleinschmldt

Prof. Dr. A. Bachem

Prot. Dr. Dr. U. Derlgs

Dr. D. Flscher

Prof. Dr. U. Leopold-Wildburger Prof. Dr. R. H. Mőhring
Anschrilt für Abstracts und weltere Informationen:

Prof. Dr. P. Kleinschmidt

Universitát Passau

Wirtschaftswissenschaftliche

Fakultăt

D-94030 Passau

Tel. +49-85T-509-2570

-mall: sor95@winf.uni-passau.de
Anschriff für dle vorläutige Tollnahmeanmeldung:

Geschäftsstelle der DGOR

Am Stelnknapp 14b

D-44795 Bochum

Tel. +49-234-462246 